\title{
Single-Incision Laparoscopic Liver Resection in Ruptured Liver Adenoma
}

\author{
Simon Wabitsch, PD Dr. med. Timm Denecke, PD Dr. med. Dominik Geisel, \\ Dr. med. Ann-Christin von Brünneck, Dr. med. Andreas Andreou, Prof. Dr. med. Matthias Biebl, \\ Prof. Dr. med. Johann Pratschke, PD Dr. med. Moritz Schmelzle \\ Campus Virchow-Klinikum, Charité Universitätsmedizin Berlin, Berlin, Germany (all authors).
}

\begin{abstract}
Introduction: Spontaneous rupture of liver tumors, such as hepatocellular adenoma (HCA) and carcinoma (HCC), is rare, but may be life threatening, as unspecific symptoms can be misleading. Immediate diagnosis, angiographic intervention and surgery is needed to stop the bleeding.

Case Description: We present a case of a ruptured HCA, which was addressed by a 2-step interventional and laparoscopic approach. A female 53-year-old patient was admitted to the hospital with unspecific epigastric pain for the past 3 days. Acute coronary syndrome was initially suggested because of a history of hypertension, but could be ruled out in further investigation. Ultrasonography and a subsequent contrast-enhanced computed tomographic (CT) scan of the abdomen showed a massive deterioration of the left-lateral section, with active bleeding from a ruptured liver tumor and free-floating blood in the peritoneal cavity. Successful emergency angiographic embolization was accomplished with gelatin foam powder (Gelita-Spon, Gelita Medical, Germany) in the hemodynamically stable patient. Subsequent magnetic imaging (MRI) with the hepatospecific contrast agent gadoxetate sodium (Gd-EOB or Primovist/Eovist; Bayer, Leverkusen, Germany) revealed the most likely diagnosis of an HCA, which had been ruptured. Two additional adenomas $<3 \mathrm{~cm}$ in diameter in liver segments 4 and 6 were diagnosed without the need for further treatment. We performed a single-incision laparoscopy to evacuate the hematoma and to address the ruptured liver tumor. Anatomic left lateral sectionectomy was performed with a harmonic scalpel and a vascular stapler. The resected liver lobe was removed through the umbilical single-port incision. The postoperative course was uneventful, and the patient was discharged on postoperative day 5 .
\end{abstract}

Discussion: This case emphasizes the importance of interdisciplinary management of ruptured liver tumors and highlights advantages of single-incision laparoscopy over laparotomy in the management of emergency liver resection.

Key Words: Angiographic intervention, Emergency laparoscopic liver resection, Ruptured liver adenoma.

Citation Wabitsch S, Denecke T, Geisel D, von Brünneck A-C, Andreou A, Biebl M, Pratschke J, Schmelzle M. Single-Incision laparoscopic liver resection in ruptured liver adenoma. CRSLS e2017.00003. DOI: 10.4293/CRSLS.2017.00003.

Copyright $\odot 2017$ by SLS, Society of Laparoendoscopic Surgeons. This is an open-access article distributed under the terms of the Creative Commons Attribution-Noncommercial-ShareAlike 3.0 Unported license, which permits unrestricted noncommercial use, distribution, and reproduction in any medium, provided the original author and source are credited.

Disclosure : none.

Address correspondence to: Simon Wabitsch, MD, Campus Virchow-Klinikum, Charite Universitatsmedizin Berlin, Augustenburgerplatz 1, 13353 Berlin, Germany. Telephone: 004930450652437, Fax: 004930450552900, E-mail: simon.wabitsch@charite.de

\section{INTRODUCTION}

Spontaneous liver bleeding occurs as a relatively rare but typical complication of hypervascularized benign and malignant tumors in the liver or can be caused by anticoagulation. $^{1-3}$ The cause of active bleeding varies, depending on the regional prevalence of hepatocellular adenoma (HCA) and carcinoma (HCC). Although, HCA is the third most common benign tumor of the liver, after liver hemangioma and focal-nodular hyperplasia, ${ }^{4} \mathrm{HCA}$ is responsible for $\sim 40 \%$ of all such cases. ${ }^{1,2}$

HCA is more common in women than in men, and the incidence in women of child-bearing age is 3 to 4 per $100,000.5$ The occurrence is related to the use of contraceptive pills, and accordingly, the incidence has been 
increasing since the 1960s. ${ }^{6,7}$ HCA can be divided into 4 subgroups: (1) HNF1a-positive adenoma, which frequently occurs in women with adenomatosis, associated with a relatively low risk of malignant transformation and bleeding; (2) inflammatory type adenoma, which occurs mostly in obese women and is characterized by a high risk of bleeding; (3) $\beta$-catenin-activated adenoma, which occurs mostly in men and is associated with the highest risk of malignancy among these 4 subgroups; and (4) nonspecific, histologically unclassifiable liver adenoma. ${ }^{7}$

Risk of spontaneous rupture with active bleeding varies between 30 and 50\% for lesions with a diameter of more than $5 \mathrm{~cm} .{ }^{8-10}$ Nonspecific symptoms of tumor bleeding make diagnosis difficult, so interdisciplinary cooperation is especially important, to be able to start the necessary treatment quickly. ${ }^{11}$ Arterial bleeding may be treated minimally invasively by angiographic embolization, followed by surgical intervention to evacuate the hematoma and remove the ruptured liver tumor. ${ }^{12}$

Herein, we report on a patient with a ruptured and actively bleeding HCA located in the left-lateral liver. This case highlights the importance of interdisciplinary management of ruptured liver tumors and advantages of single-incision laparoscopy over laparotomy in the management of emergency liver resection.

\section{Case Description}

A 53-year-old woman presented with severe pain in the epigastric region for the past 3 days. Because of her medical history of hypertension, acute coronary syndrome was suspected, and $5000 \mathrm{IU}$ heparin and $500 \mathrm{mg}$ aspirin were administered. Electrocardiogram and troponin levels were found to be normal, but hemoglobin level had decreased to $7.7 \mathrm{mg} / \mathrm{dL}$. A sonogram and subsequent computed tomographic (CT) scan of the abdomen showed free abdominal fluid and active bleeding from a lesion in the left lateral liver section. The patient immediately received $1 \mathrm{~g}$ tranexamic acid, $16 \mu \mathrm{g}$ desmopressin, $4 \mathrm{~g}$ fibrinogen, 2 units of erythrocyte concentrate, and 4 units of fresh frozen plasma and was referred to our hospital for further treatment.

The initial hemoglobin levels at admission were $7.3 \mathrm{~g} / \mathrm{dL}$. Emergency angiography was indicated to address the active arterial bleeding and was performed by means of temporary embolization with gelatin foam powder (Gelita-Spon; Gelita Medical) of 2 accessory segmental arteries from the left gastric artery (Figure 1). Platelets were transfused to address decreased thrombocyte counts of $86 / \mathrm{nL}$. A postinterventional CT showed an extensive
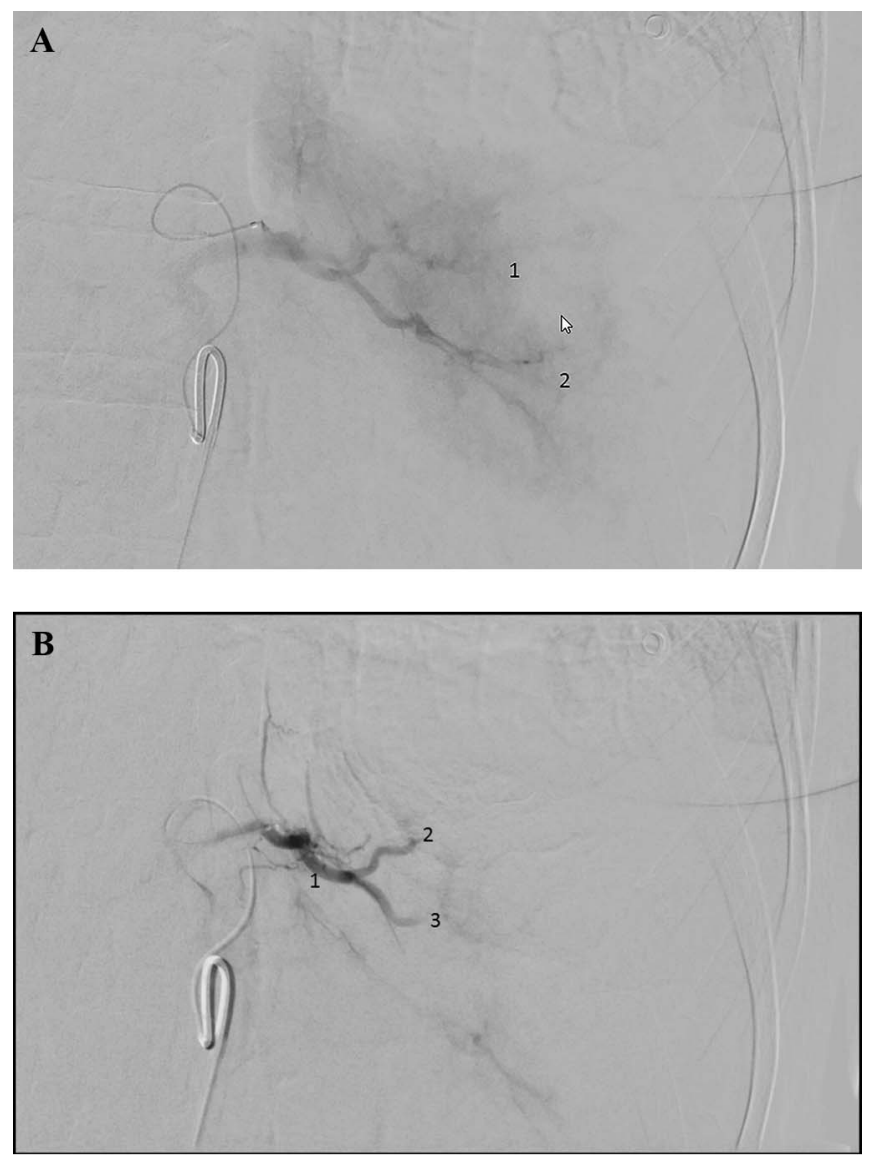

Figure 1. Angiographic embolization. A, 1, 2: bleeding segment of artery. B, 1: left accessory liver artery; 2, 3: segment artery embolization with gelatin.

intraperitoneal hematoma, as well as a successfully performed embolization of the tumorous lesion. The patient was transferred to the surgical intensive care unit, where her hemoglobin levels remained stable.

The entity of the ruptured lesion in the left-lateral section could not be discerned from the multiphasic preinterventional CT. In addition, 2 small hypervascularized lesions were suspected in liver segments 4 and 6. For further characterization of liver lesions in a now hemodynamically stable patient, an MRI of the abdomen was performed with gadoxetate (Gd-EOB; Bayer) used as hepatocyte specific contrast agent. ${ }^{13}$ MRI raised a suspicion of HCA as the most likely diagnosis in the absence of venous contrast washout and the absence of intracellular late enhancement with a diameter of 12.5 in the left-lateral section. Other tumors in segments $4 \mathrm{~b}, 5$, and 8 were also diagnosed as HCA by MRI with tumors of a diameter $<3 \mathrm{~cm}$ and no further surgical intervention needed (Figure 2). A 



Figure 2. Preoperative MRI. A, 1: Adenoma with diameters of 12.5 and $9.5 \mathrm{~cm}$; : free abdominal fluid. B, 1: Adenoma with a diameter of $1.8 \mathrm{~cm}$ in segment 4 . C, 1 , adenoma with a diameter of $2.5 \mathrm{~cm}$ in segment 5 .

laparoscopic exploration was planned to evacuate the hematoma and to perform an anatomic left-lateral sectionectomy to remove the ruptured HCA.

We made a single incision at the umbilicus to insert a wound retractor (Alexis O; Applied Medical, Middletown, New York, USA). In addition, a size 12 trocar (Kii Optical; Applied Medical) was installed at the left epigastrium, which was later used for wound drainage. A capnoperitoneum and camera (Olympus, Hauppauge, New York, USA) were inserted (Figure 3).
In the abdomen, a large amount of partially coagulated blood was found, which was removed by suction. The round and falciform ligaments were intersected, and the left triangular ligament was released centrally. The lesser omentum was not opened. Superficial transection was performed along the falciform ligament with a harmonic scalpel (Harmonic Ace; Ethicon, Somerville, New Jersey, USA), and bipolar coagulation was performed repeatedly. Transection of deeper tissue and central structures was performed with 3 magazines staple cartridges of a vascular 


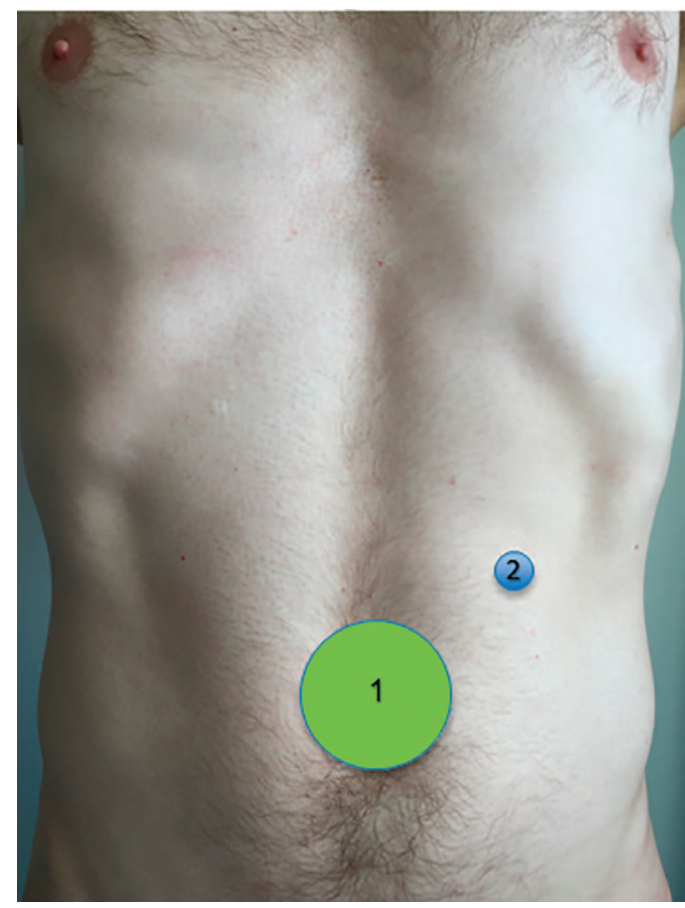

Figure 3. Pattern of incisions. Umbilical incision 1: wound retractor; 2 12-mm trocar.

Echelon Endopath stapler (Ethicon). Finally, the left triangular ligament was completely released. The resected specimen was retrieved transumbilically by means of an Espiner Master E-Sac 1800 mL (Espiner Medical Limited, Clevedon, United Kingdom) (Figure 4).

Again, bipolar and infrared coagulation was performed. The operation was performed in 148 minutes.

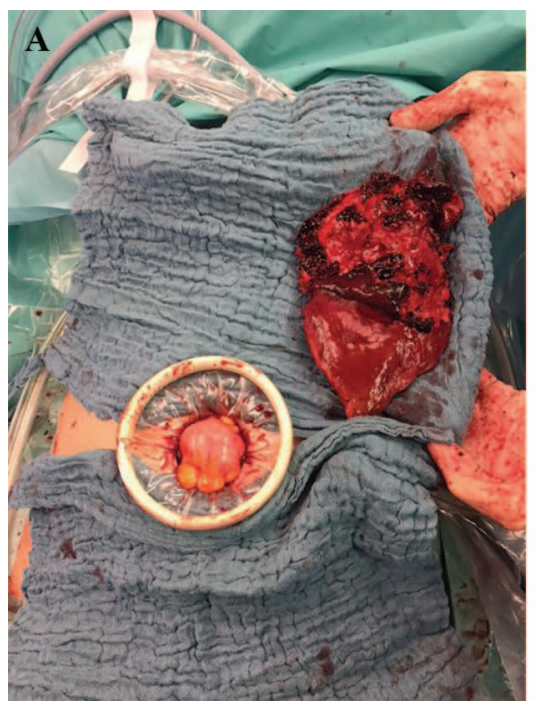

Figure 4. Single port and resected segments 2 and 3 .
The postoperative course of the patient was uneventful. The patient was transferred to the surgical ward on postoperative day (POD) 1. Stepwise transition to a normal diet, mobilization, and wound healing were unremarkable at all times. The wound drain was removed on POD 4 and the patient was released on POD 5. Histopathologic examination confirmed a ruptured HCA without any evidence of malignant transformation (Figure 5). The patient was seen in our outpatient clinic 1 week after discharge to discuss further management. We recommended that the remaining HCAs in the right lobe of the liver be followed up in 3 months with diagnostic imaging. The oral contraceptive medication had been stopped by the patient on the first day of the symptoms, and we recommended that it not be reinstituted.

\section{DISCUSSION}

In case of suspected active arterial bleeding in the CT scan, emergency angiography and angioembolization is considered to be the initial therapy of choice. ${ }^{14,15} \mathrm{Al}$ though, laparoscopy is associated with well-known benefits (eg, decreased blood loss, less operative trauma, and a shorter hospital stay), ${ }^{16}$ laparotomy and open liver surgery remain the standard approach at most institutions in emergency settings. ${ }^{17}$

We report on a patient, who was referred to our hospital with an actively bleeding adenoma in the left-lateral liver section. We performed a 2-stage procedure with angioembolization of segmental left-lateral arteries and subsequent single-incision laparoscopic left lateral sectionec-

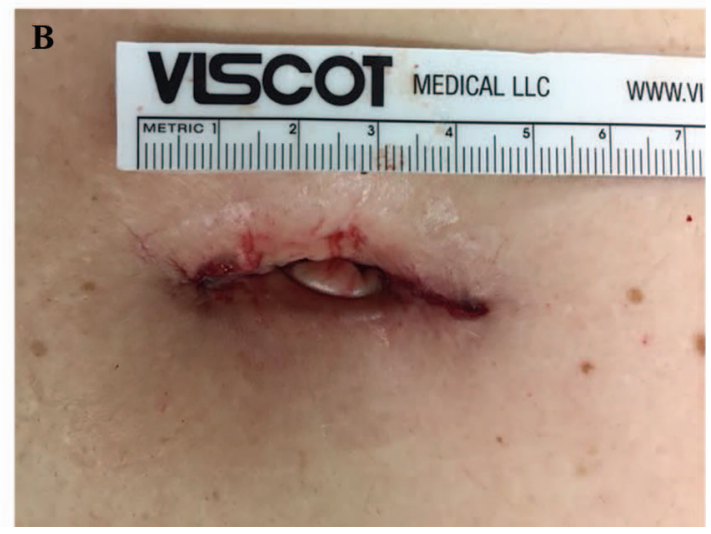




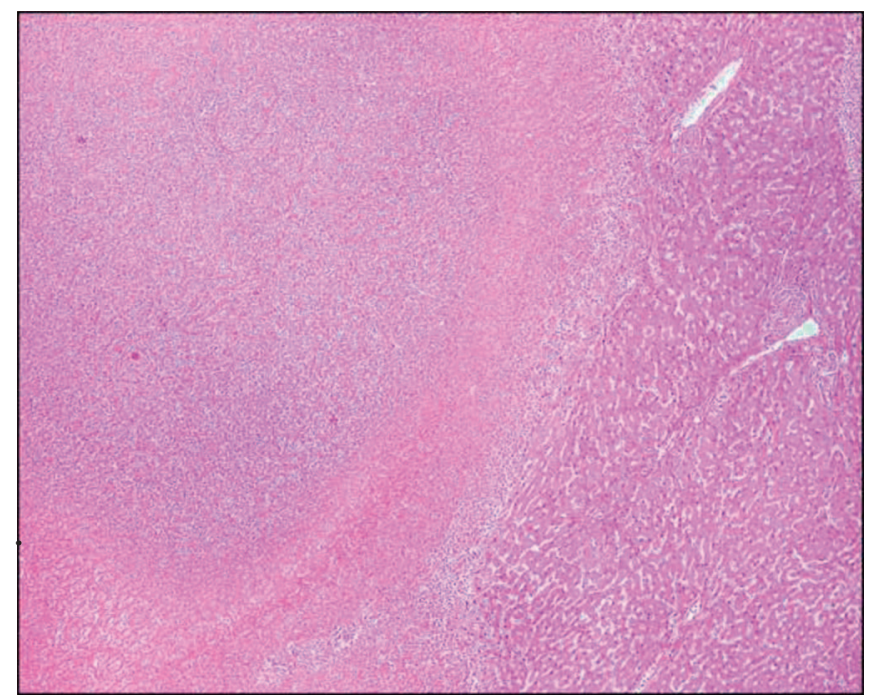

Figure 5. Histopathologic examination.

tomy with evacuation of the intraperitoneal hematoma. The postoperative course was uneventful, and the patient was discharged at POD 5.

The 2-step minimally invasive surgical approach became possible because of efficient embolization that stabilized the patient, and stopped the bleeding and prevented a recurrence. Even if embolization was not effective, the necessary operation would be started laparoscopically, as it allows a good overview of the intraabdominal situation, and if necessary, more invasive approaches can be planned appropriately.

Laparoscopy made it safe to use the most reliable noninvasive diagnostic modality, MRI with hepatocyte-specific contrast, to be more secure about the entity of the lesion responsible for the bleeding. ${ }^{18,19}$ With the high probability of a benign lesion to be resected at the site of rupture and the additional lesions without an immediate indication for surgical removal, it was safe to perform the resection laparoscopically instead of an open resection.

In our opinion, the benefits of routine laparoscopic liver surgery, which should also be taken into account in emergency settings, are:

For left-lateral sectionectomy, this approach provides a very good view and ideal angle of instruments for the region in question.

Laparoscopic surgery gives a better overview when the location of the bleeding is not obvious.

The incision can be easily extended when needed for further exploration, or a hand port can be inserted.
The approach also allows good access to other locations such as the sigmoid or appendix.

The operation time is an important factor especially in an emergency situation. This approach allows a quicker exploration, and an additional incision to recover the resected lobe from the abdomen is not needed.

Smaller abdominal trauma leads to reduction of postoperative pain and quicker mobilization and thus a shorter hospital stay. ${ }^{20-22}$

A report of reduced intraoperative blood loss, a better cosmetic result, and reduced recovery time in the hospital for patients with a single-port approach have recently been published. ${ }^{23}$

One limitation of single-port laparoscopy is the limited access to the hepatocaval confluence, and this drawback can be a problem when extended liver surgery is needed. However, in case of limited triangulation, additional trocars can be inserted.

Single-incision laparoscopic left-lateral sectionectomy is technically feasible and safe, even in emergency settings. Prospective studies are urgently needed to investigate whether patients benefit more from single-incision laparoscopic left lateral sectionectomy, when compared to multiport approaches.

\section{References:}

1. Battula N, Tsapralis D, Takhar A, et al. Aetio-pathogenesis and the management of spontaneous liver bleeding in the West: a 16-year single-centre experience. HPB. 2012;14:382-389.

2. Dewar GA, Griffin SM, Ku KW, Lau WY, Li AK. Management of bleeding liver tumours in Hong Kong. Br J Surg. 1991;78:463466.

3. Freyhardt P, Seehofer D, Denecke T. Spontaneous pronounced intrahepatic hematoma during oral anticoagulation (in German). Röfo Pogress in the subject $x$-ray and nukl. medicine 2011;183:1066-1068.

4. Margonis GA, Ejaz A, Spolverato G, et al. Benign solid tumors of the liver: management in the modern era. J Gastrointest Surg. 2015;19:1157-1168.

5. Rooks JB, Ory HW, Ishak KG, et al. Epidemiology of hepatocellular adenoma: the role of oral contraceptive use. JAMA. 1979;242:644-648.

6. Baum J, Bookstein J, Holtz F, Klein E. Possible association between benign hepatomas and oral contraceptives. Lancet. 1973;302:926-929.

7. Klatskin G. Hepatic tumors: possible relationship to use of oral contraceptives. Gastroenterology. 1977;73:386-394. 
8. Zucman-Rossi J, Jeannot E, Nhieu JTV, et al. Genotypephenotype correlation in hepatocellular adenoma: new classification and relationship with HCC. Hepatology. 2006;43:515-524.

9. Gibbs JF, Litwin AM, Kahlenberg MS. Contemporary management of benign liver tumors. Surg Clin North Am. 2004;84: $463-480$.

10. Leese T, Farges O, Bismuth H. Liver cell adenomas: a 12-year surgical experience from a specialist hepato-biliary unit. Ann Surg. 1988;208:558-564.

11. Srinivasa S, Lee WG, Aldameh A, Koea JB. Spontaneous hepatic haemorrhage: a review of pathogenesis, aetiology, and treatment. $H P B$. 2015;17:872-880.

12. Zhu LX, Wang GS, Fan ST. Spontaneous rupture of hepatocellular carcinoma. Br J Surg. 1996;83:602-607.

13. Grieser C, Denecke T, Rothe J-H, et al. Gd-EOB enhanced MRI T1-weighted 3D-GRE with and without elevated flip angle modulation for threshold-based liver volume segmentation. Acta Radiol. 2015;56:1419-1427.

14. Battula N, Madanur M, Priest O, et al. Spontaneous rupture of hepatocellular carcinoma: a Western experience. Am J Surg. 2009; 197:164-167.

15. Matthaei H, Boelke E, Eisenberger CF, et al. Interdisciplinary treatment of primary hepatic angiosarcoma: emergency tumor embolization followed by elective surgery. Eur J Med Res. 2007; 12:591-594.

16. Nguyen KT, Gamblin TC, Geller DA. World review of laparoscopic liver resection: 2,804 patients. Ann Surg. 2009;250:831-841.
17. Tanaka A, Takeda R, Mukaihara S, et al. Treatment of ruptured hepatocellular carcinoma. Int J Clin Oncol. 2001;6:291295.

18. Grazioli L, Bondioni MP, Haradome H, et al. Hepatocellular adenoma and focal nodular hyperplasia: value of gadoxetic acid-enhanced MR imaging in differential diagnosis. Radiology. 2012;262:520-529.

19. Grieser C, Steffen IG, Kramme I-B, et al. Gadoxetic acid enhanced MRI for differentiation of FNH and HCA: a single centre experience. Eur Radiol. 2014;24:1339-1348.

20. Shetty GS, You YK, Choi HJ, Na GH, Hong TH, Kim D-G. Extending the limitations of liver surgery: outcomes of initial human experience in a high-volume center performing singleport laparoscopic liver resection for hepatocellular carcinoma. Surg Endosc. 2012;26:1602-1608.

21. Tayar C, Claude T, Subar D, et al. Single incision laparoscopic hepatectomy: advances in laparoscopic liver surgery. $J$ Minim Access Surg. 2014;10:14-17.

22. Chang SKY, Mayasari M, Ganpathi IS, Wen VLT, Madhavan K. Single port laparoscopic liver resection for hepatocellular carcinoma: a preliminary report. Int J Hepatol. 2011; 2011:579203.

23. Benzing C, Krenzien F, Atanasov G, et al. Single incision laparoscopic liver resection (SILL): a systematic review. GMS Interdiscip Plast Reconstr Surg DGPW. In press. 